TABLE DR1. (U-Th)/He DATA

\begin{tabular}{|c|c|c|c|c|c|c|c|c|c|c|c|c|c|}
\hline $\begin{array}{l}\text { Local } \\
\text { sample no. }\end{array}$ & Age/Formation & Rock type & $\begin{array}{c}\text { Elev. } \\
(\mathrm{m})\end{array}$ & $\begin{array}{l}\text { UTM } \\
\text { (east) }\end{array}$ & $\begin{array}{c}\text { UTM } \\
\text { (north) }\end{array}$ & Segment & $\begin{array}{c}\mathrm{U} \\
(\mathrm{ppm})\end{array}$ & $\begin{array}{c}\mathrm{Th} \\
(\mathrm{ppm})\end{array}$ & $\begin{array}{c}\mathrm{He} \\
(\mathrm{nmol} / \mathrm{g})\end{array}$ & $\mathrm{Ft}^{\#}$ & $\begin{array}{c}\text { Corrected He } \\
\text { age }(\mathrm{Ma})\end{array}$ & $\begin{array}{c}\text { Average He } \\
\text { age (Ma) }\end{array}$ & $\begin{array}{l}\text { Uncert. } \\
(\mathrm{Ma})^{\# \#}\end{array}$ \\
\hline AT00-02-1 & Precamb. & Gneiss & 1445 & 414695 & 4584913 & Brigham & 3.391 & 0.556 & 0.055 & 0.705 & 4.416 & & \\
\hline AT00-02-2 & Farmington & & 1445 & 414695 & 4584913 & Brigham & 7.844 & 0.318 & 0.132 & 0.703 & 4.464 & & \\
\hline AT00-02-3 & Canyon & & 1445 & 414695 & 4584913 & Brigham & 8.077 & 0.547 & 0.120 & 0.709 & 3.807 & & \\
\hline AT00-02-4 & & & 1445 & 414695 & 4584913 & Brigham & 11.052 & 1.362 & 0.236 & 0.669 & 5.699 & 4.6 & 0.8 \\
\hline AT00-03-1 & Precamb. & Granitic & 1535 & 414976 & 4580965 & Brigham & 17.733 & 4.520 & 0.269 & 0.710 & 3.703 & & \\
\hline AT00-03-2 & Farmington & gneiss & 1535 & 414976 & 4580965 & Brigham & 18.027 & 3.001 & 0.295 & 0.703 & 4.125 & & \\
\hline АТ00-03-4 & Canyon & & 1535 & 414976 & 4580965 & Brigham & 27.941 & 4.685 & 0.445 & 0.650 & 4.336 & & \\
\hline AT00-03-5 & & & 1535 & 414976 & 4580965 & Brigham & 21.820 & 5.427 & 0.319 & 0.694 & 3.657 & & \\
\hline AT00-03-6 & & & 1535 & 414976 & 4580965 & Brigham & 22.441 & 7.104 & 0.642 & 0.710 & 6.906 & 4.5 & 1.4 \\
\hline AT00-13-1 & Precamb. & Granitic & 1450 & 420980 & 4568510 & Weber & 67.784 & 47.578 & 0.965 & 0.673 & 3.342 & & \\
\hline AT00-13-2 & Farmington & gneiss & 1450 & 420980 & 4568510 & Weber & 32.445 & 23.264 & 0.524 & 0.686 & 3.711 & & \\
\hline AT00-13-3 & Canyon & & 1450 & 420980 & 4568510 & Weber & 18.728 & 9.675 & 0.312 & 0.674 & 4.064 & & \\
\hline AT00-13-4 & & & 1450 & 420980 & 4568510 & Weber & 50.265 & 33.431 & 0.889 & 0.695 & 4.050 & 3.8 & 0.3 \\
\hline AT00-15-2 & Precamb. & Granitic & 1745 & 422202 & 4559614 & Weber & 79.410 & 28.388 & 1.829 & 0.661 & 5.914 & & \\
\hline AT00-15-3 & $\begin{array}{l}\text { Farmington } \\
\text { Canyon }\end{array}$ & gneiss & 1745 & 422202 & 4559614 & Weber & 88.639 & 30.544 & 2.073 & 0.648 & 6.146 & 6.0 & 0.2 \\
\hline WEB99-17-1 & Precamb. & Granitic & 1430 & 424685 & 4554000 & Weber & 35.147 & 5.874 & 0.985 & 0.663 & 7.488 & & \\
\hline WEB99-17-2 & $\begin{array}{l}\text { Farmington } \\
\text { Canyon }\end{array}$ & gneiss & 1430 & 424685 & 4554000 & Weber & 96.605 & 26.445 & 1.971 & 0.673 & 5.242 & 6.4 & 1.6 \\
\hline WEB99-15-2 & Precamb. & Amphibolit $\epsilon$ & 1450 & 426866 & 4531912 & Weber & 17.372 & 0.000 & 0.292 & 0.664 & 4.654 & & \\
\hline WEB99-15-3 & Farmington & gneiss & 1450 & 426866 & 4531912 & Weber & 13.653 & 1.246 & 0.200 & 0.669 & 3.944 & & \\
\hline WEB99-15-4 & Canyon & & 1450 & 426866 & 4531912 & Weber & 25.515 & 3.688 & 0.294 & 0.633 & 3.244 & 3.9 & 0.7 \\
\hline AT00-18-1 & Tertiary & Volcanic & 1450 & 426157 & 4516196 & Salt Lake & 2.781 & 16.419 & 0.886 & 0.796 & 30.789 & & \\
\hline AT00-18-2 & & breccia & 1450 & 426157 & 4516196 & Salt Lake & 9.736 & 33.688 & 2.860 & 0.780 & 38.130 & & \\
\hline AT00-18-3 & & & 1450 & 426157 & 4516196 & Salt Lake & 8.985 & 52.823 & 3.620 & 0.763 & 40.712 & 37 & 5.1 \\
\hline RB-02-1 & Triassic & Sandstone & 1610 & 430750 & 4513150 & Salt Lake & 1.611 & 13.093 & 0.126 & 0.690 & 7.173 & & \\
\hline RB-02-2 & Ankareh Fm & & 1610 & 430750 & 4513150 & Salt Lake & 16.665 & 40.867 & 0.368 & 0.678 & 3.802 & & \\
\hline RB-02-3 & & & 1610 & 430750 & 4513150 & Salt Lake & 13.558 & 79.309 & 0.727 & 0.694 & 5.978 & & \\
\hline RB-02-4 & & & 1610 & 430750 & 4513150 & Salt Lake & 7.642 & 23.663 & 0.283 & 0.685 & 5.752 & 5.7 & 1.4 \\
\hline $9808(1)^{*}$ & Tertiary LCS & QM & 1780 & 434250 & 4495950 & Salt Lake & 7.400 & 9.000 & 0.217 & 0.780 & 5.300 & & \\
\hline $9808(2)^{*}$ & & & 1780 & 434250 & 4495950 & Salt Lake & 6.900 & 7.500 & 0.256 & 0.760 & 7.200 & 6.2 & 1.3 \\
\hline
\end{tabular}




\begin{tabular}{|c|c|c|c|c|c|c|c|c|c|c|c|c|c|}
\hline $\begin{array}{l}\text { Local } \\
\text { sample no. }\end{array}$ & Age/Formation & Rock type & $\begin{array}{l}\text { Elev. } \\
(\mathrm{m})\end{array}$ & $\begin{array}{l}\text { UTM } \\
\text { (east) }\end{array}$ & $\begin{array}{c}\text { UTM } \\
\text { (north) }\end{array}$ & Segment & $\begin{array}{c}\mathrm{U} \\
(\mathrm{ppm})\end{array}$ & $\begin{array}{c}\text { Th } \\
(\mathrm{ppm})\end{array}$ & $\begin{array}{c}\mathrm{He} \\
(\mathrm{nmol} / \mathrm{g})\end{array}$ & $\mathrm{Ft}^{\#}$ & $\begin{array}{c}\text { Corrected He } \\
\text { age (Ma) }\end{array}$ & $\begin{array}{c}\text { Average } \mathrm{He} \\
\text { age (Ma) }\end{array}$ & $\begin{array}{l}\text { Uncert. } \\
(\mathrm{Ma})^{\# \#}\end{array}$ \\
\hline $9810(2)^{*}$ & & & 2390 & 435100 & 4494700 & Salt Lake & 9.800 & 23.900 & 0.320 & 0.750 & 5.100 & 4.7 & 0.9 \\
\hline LC-2* & Tertiary LCS & QM & 1520 & 429250 & 4485000 & Salt Lake & 12.900 & 24.800 & 0.179 & 0.730 & 1.600 & 1.6 & 0.2 \\
\hline $\mathrm{LC}-5^{*}$ & Tertiary LCS & QM & 2100 & 430800 & 4484380 & Salt Lake & 13.200 & 25.300 & 0.229 & 0.770 & 2.600 & 2.6 & 0.4 \\
\hline AT00-06-4 & Tillite & from tillite & 1590 & 446930 & 4457246 & Provo & 12.479 & 21.798 & 0.503 & 0.667 & 7.873 & & \\
\hline AT00-07-2 & & & 1590 & 446930 & 4457246 & Provo & 7.914 & 10.897 & 0.239 & 0.790 & 5.307 & 6.4 & 1.2 \\
\hline AT00-10-1 & Precamb. & Granitic & 1850 & 435523 & 4422864 & Nephi & 3.724 & 14.938 & 0.211 & 0.702 & 7.629 & & \\
\hline AT00-10-2 & $\begin{array}{l}\text { Farmington } \\
\text { Canyon }\end{array}$ & gneiss & 1850 & 435523 & 4422864 & Nephi & 5.570 & 15.857 & 0.178 & 0.668 & 5.256 & 6.4 & 1.7 \\
\hline
\end{tabular}

Notes:

* Multiple-grain furnace (U-Th)/He ages from Armstrong et al. (2003). All others are single-grain ages (House et al., 2000).

\# Ft is alpha-stopping distance correction factor (Farley et al., 1996).

\#\# Uncertainty is $1 \sigma ; 15 \%$ for LC-2 and LC-5 (Armstrong et al., 2003).

\section{Other notes:}

Dashed numbers in the Local sample no. designation are individual grains for that sample separate. LCS is Little Cottonwood Stock. QM is quartz monzonite. Numbers in parentheses for sample no. are replicate numbers for multiple-grain furnace ages.

Apatite crystals were inspected under cross-polarized light at 110X power to avoid U-bearing inclusions and visible defects. Grain lengths and widths were measured on 2 to 5 grains for each sample. Average grain width is $100 \mu \mathrm{m}$ with a range of $72-140 \mu \mathrm{m}$. He ages were corrected for $\alpha$-emission effects (Farley et al., 1996) and then averaged for each sample to give a mean He age. Uncertainties are at $1 \sigma$ and are based on single grain age distributions for each sample or on the repeatability of multiple-grain furnace He ages.

Both single-grain laser ages and multiple-grain furnace ages were measured in the Helium lab at Caltech, run by Ken Farley. Consistency between single-grain ages for a particular sample provide confidence that the grains do not contain uranium-rich inclusions, such as zircon, that would produce excess amounts of helium relative to samples with no inclusions (Ehlers and Farley, 2003). For multiple-grain ages (whereby several grains are essentially homogenized), unknown inclusions could lead to excess helium and then an interpretation of an older apparent age. Thus, multiple-grain samples LC-2 and LC-5, which do not have replicate analyses, should be considered maximum ages. The other two multiple-grain samples 9808 and 9810 have replicate analyses that show age consistency and provide age confidence.

\section{References Cited}

Armstrong, P.A., Ehlers, T.A., Chapman, D.S., Farley, K.A., and Kamp, P.J.J., 2003, Exhumation of the central Wasatch Mountains, 1: Patterns and timing deduced from low-temperature thermochronometry data: Journal of Geophysical Research, v. 108, no. B3, 2172, doi:10.1029/2001JB001708.

Ehlers, T.A., and Farley, K.A., 2003, Apatite (U-Th)/He thermochronometry: methods and applications to problems in tectonic and surface processes: Earth and Planetary Science Letters, v. 206, p. 1-14.

Farley, K.A., Wolf, R.A., and Silver, L.T., 1996, The effects of long alpha-stopping distances on (U-Th)/He ages: Geochimica et Cosmochimica Acta, v. 60, p. 4223-4229.

House, M.A., Farley, K.A., and Stockli, D., 2000, Helium chronometry of apatite and titanite using Nd-YAG laser heating: Earth and Planetary Science Letters, v. 183 , p. $365-368$. 